\title{
KHẢO SÁT Độ CHÍNH XÁC CÔNG NGHỆ TRẠM CORS TRONG MộT SỐ DẠNG CÔNG TÁC TRĂC ĐỊA Độ CHÍNH XÁC CAO
}

\author{
TRẦ VIẾT TUẤN ${ }^{(1)}$, NGUYẼ̃N VĂN HIỆP(2)

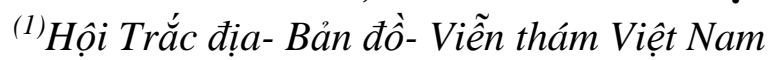 \\ ${ }^{(2)}$ Công ty TNHH khảo sát và xây dựngViệt Mỹ
}

\section{Tóm tắt:}

Nội dung của bài báo trình bày về kết quả nghiên cưu khảo sát độ chính xác đạt được của công nghệ trạm cors khi định vị điểm đon trong điều kiện Việt Nam. Co sở lý thuyết của phuong pháp khảo sát, kết quả đo đạc thưc nghiệm và đánh giá khả năng ứng dụng của công nghệ tiên tiến này trong một số dạng công tác trắc địa yêu cầu độ chính xác cao ở Việt Nam

\section{1. Đặt vấn đề}

Hiện nay trong công tác trắc địa có một số dạng công việc đòi hỏi phải xác định được toạ độ tức thời của một số điểm đặc biệt trên khu vực theo thời gian thực nhằm mục đích thực hiện các nội dung chuyên môn phục vụ thi công xây dựng hoặc theo dõi sự ổn định của địa hình hoặc các công trình xây dựng bị ảnh hưởng do tác động của hiện tượng biến đổi khí hậu gây ra. Có thể lấy một số ví dụ điển hình sau đây:

- Theo dõi và cảnh báo các hiện tượng trượt lở đồi núi tại khu vực miền núi phía Bắc, miền Trung do ảnh hưởng của mưa lũ gây ra.

- Theo dõi và cảnh bảo hiện tượng trượt lở tại các tuyến sông rạch vùng đồng bằng sông Cửu Long do ảnh hưởng của hiện tượng biến đổi khí hậu gây ra ở Việt Nam.v.v

Vấn đề cần đặt ra nghiên cứu ở đây là: Cần phải lựa chọn công nghệ, thiết bị hiện đại đang có tại Việt Nam có độ chính xác đáp ứng được các yêu cầu cần thiết đối với từng dạng công tác trắc địa cần theo dõi liên tục theo thời gian thực trong một khoảng thời gian dài với độ chính xác cao. Ở nước ta công nghệ trạm CORS đã được nghiên cứu và ứng dụng tương đối rộng rãi trong nhiều lĩnh vực của nền kinh tế quốc dân. Đây là dạng công nghệ tiên tiến và có thể đáp ứng được các yêu cầu kỹ thuật theo dõi và cảnh báo các hiện tượng trượt lở đất đá do ảnh hưởng của biến đổi khí hậu gây ra. Tuy nhiên để có cơ sở khoa học sử dụng công nghệ này trong công tác theo dõi và cảnh bảo các hiện tượng bất thường của địa hình do ảnh hưởng biến đổi khí hậu gây ra ở nước ta cần phải khảo sát độ chính xác đạt được của dạng công nghệ trạm CORS trong điều kiện Việt Nam

\section{Cơ sở lý thuyết và phương pháp nghiên cứu \\ 2.1. Công nghệ trạm tham chiếu liên tục CORS}

Trạm CORS (Continuously Operating Reference Station) là các trạm GNSS cố định trong đó lắp đặt máy thu tín hiệu GNSS trên mốc cố định được xác định chính xác tọa độ và có khả năng thu tín hiệu vệ tinh GNSS liên tục 24 giờ trong một ngày. Một nhóm các trạm CORS được liên kết hình thành mạng lưới các trạm CORS. Hệ thống trạm CORS bao gồm các trạm tham chiếu làm việc liên tục, cố định, sủ dụng công nghệ máy tính hiện đại và internet truyền dữ liệu tạo thành một mạng lưới. Do có nhiều thông tin từ nhiều trạm tham chiếu truyền tới nên tại trạm chủ, người ta có thể xây dựng được mô hình số cải chính vi phân tức thời như là hàm của vị trí

Ngày nhận bài: 25/8/2021, ngày chuyển phản biện: 1/9/2021, ngày chấp nhận phản biện: 5/9/2021, ngày chấp nhận đăng: 8/9/021 
điểm các trạm tham chiếu. Mật độ các trạm tham chiếu được xây dựng tương đối đồng đều và thông thường có khoảng cách là $70 \mathrm{~km}$. Trong một số dạng công tác trắc địa công trình, sẽ sử dụng các máy thu GNSS có khả năng thu nhận tín hiệu từ trạm CORS và phần mềm xử lý tích hợp đo theo phương pháp đo động RTK hoặc xử lý sau PPK [2]

Theo một số kết quả nghiên cứu trên thế giới, công nghệ trạm CORS cho phép xác định toạ độ và độ cao của 1 điểm bất kỳ nằm cách trạm tham chiếu từ 30-40 km với độ chính xác về mặt bằng từ $1-2 \mathrm{~cm}$ và $3-4 \mathrm{~cm}$ về độ cao $[5,6]$. Gần đây bằng cách so sánh toạ độ tại một số điểm cố định

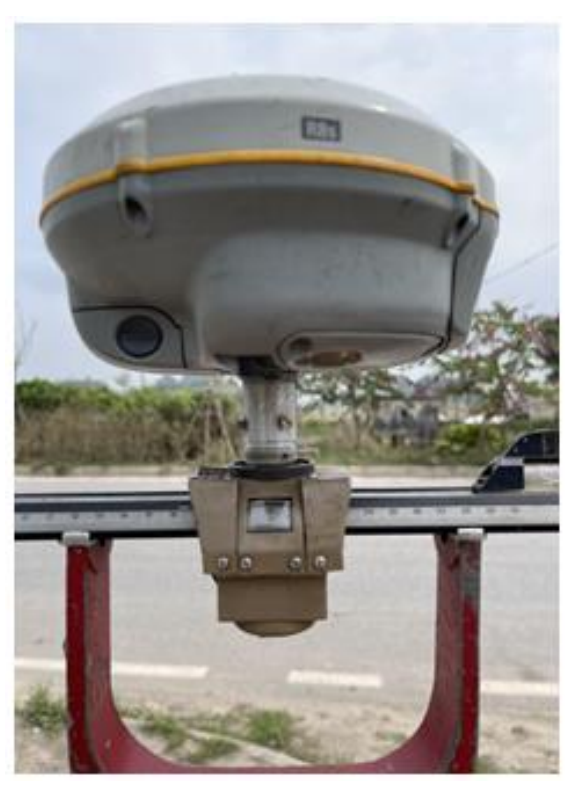

Hình 1

2.2. Khảo sát độ chính xác của công nghệ trạm CORS khi xác định tọ̣ độ điểm quan sát theo thò gian thỵc

Chúng tôi đã tiến hành đo đạc khảo sát độ chính xác định vị điểm bằng công nghệ trạm CORS tại khu vực công viên thiên văn (Dương kinh - Hà Đông) và khu vực chùa Thầy (Quốc Oai - Hà Nội) vào ngày 27/02/2021 với hệ thống máy thu của hãng Trimble: GNSS R8s [8] (hình 2). Điểm thu tín hiệu nằm cách trạm tham chiếu có khoảng cách lần lượt là $7,8 \mathrm{~km}$ và $14,4 \mathrm{~km}$. được đo theo các phuơng pháp khác nhau, các nhà khoa học Thổ Nhĩ Kỳ đã xác định được độ chính xác của công nghệ trạm CORS so với kết quả đo GPS tĩnh đạt độ chính xác về vị trí mặt bằng $\mathrm{m}_{\mathrm{P}}= \pm 0.62 \mathrm{~cm}$ và độ cao là $\mathrm{m}_{\mathrm{h}}= \pm 0.86$ cm [7]. Ở Việt Nam khi sử dụng trạm CORS đơn bố trí tại Hà Nội đo thực nghiệm tại một số điểm đã có tọa độ và độ cao Quốc gia, chúng tôi thấy độ chính xác mặt bằng đạt giá trị $\mathrm{m}_{\mathrm{P}}= \pm 2,2 \mathrm{~cm}$ $[1,2]$. Để có thể khảo sát độ chính xác của công nghệ trạm CORS đạt được khi thực hiện trong lưới trạm Network Real Time Kinemactic CORS (NRTK), chúng tôi đã tiến hành đo thực nghiệm để khảo sát độ chính xác đạt được của công nghệ trạm CORS trong điều kiện Việt Nam.

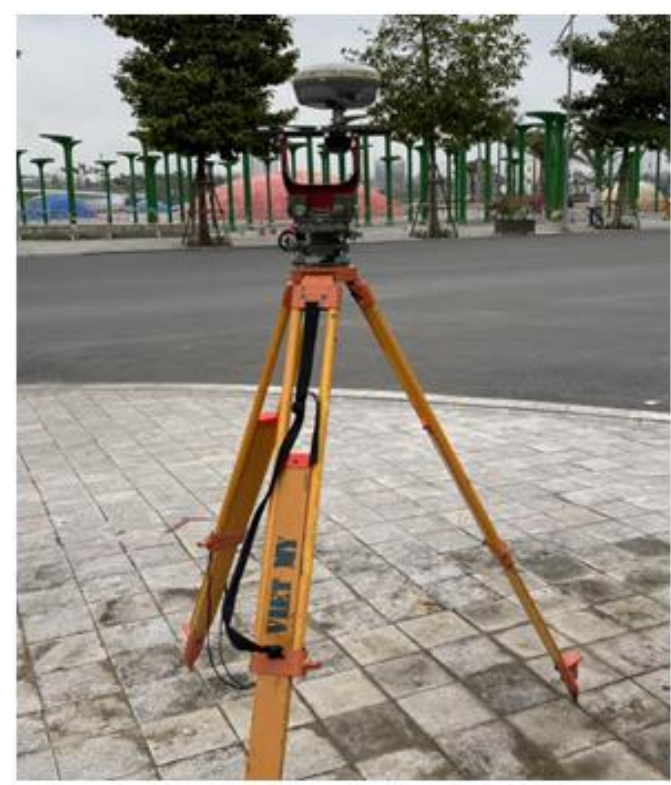

Hình 2

Để có thể xác định sai số vị trí tương hỗ giữa hai điểm đo bằng công nghệ trạm CORS, chúng tôi đã sử dụng máy thu R8s được gắn trên một thiết bị chuyên dụng: đó là một hệ thống thước chuẩn bằng thép không gỉ, trên có khắc vạch chia đến $\mathrm{mm}$, được trang bị kính hiển vi đọc số đến 0.1 $\mathrm{mm}$. Máy thu GNSS có thể chuyển động trên thước với khoảng cách tuỳ ý, và giá trị chuyển dịch được đọc trực tiếp trên thước với độ chính xác đọc số $\pm 0.1 \mathrm{~mm}$ (hình 1). 
2.2.1. Nội dung thực nghiệm tại công viên Thiên văn (Khoảng cách đến trạm tham chiếu là $7,8 \mathrm{~km}$ )

- Đầu tiên chúng tôi đặt máy thu GNSS và tiến hành thu tín hiệu trong thời gian khoảng 10 phút như hình 2.

- Tiếp theo dịch chuyển máy thu GNSS trên thước đi từng đoạn $10 \mathrm{~mm}$ tại mỗi vị trí thu tín hiệu vệ tinh khoảng 5 phút với tần suất thu tín hiệu là 1s (hình 1)

- Tiến hành xử lý kết quả đo thực nghiệm bằng cách so sánh giữa chiều dài cạnh $\mathrm{S}_{\mathrm{i}}$ đo bằng công nghệ trạm CORS và khoảng cách dịch chuyển thực tế để đánh giá độ chính xác định vị của công nghệ trạm cors theo các công thức sau đây [3]

- Tại mỗi vị trí thu tín hiệu GNSS (thứ i) tính trị trung bình của $\mathrm{k}$ trị đo GNSS

$$
\begin{aligned}
& X_{i}=\frac{1}{k} \sum_{j=1}^{j=k} x_{j} \\
& Y_{i}=\frac{1}{k} \sum_{j=1}^{j=k} y_{j}
\end{aligned}
$$

- Tính chiều dài mỗi đoạn đo theo công thức

$$
\mathrm{S}_{\mathrm{i}}=\sqrt{\Delta_{\mathrm{X}_{\mathrm{i}}}^{2}+\Delta_{\mathrm{Y}_{\mathrm{i}}}^{2}}
$$

Trong đó :

$$
\begin{aligned}
& \Delta_{\mathrm{X}_{\mathrm{i}}}=\mathrm{X}_{\mathrm{i}}-\mathrm{X}_{\mathrm{i}-1} \\
& \Delta_{\mathrm{Y}_{\mathrm{i}}}=\mathrm{Y}_{\mathrm{i}}-\mathrm{Y}_{\mathrm{i}-1}
\end{aligned}
$$

- Tính độ lệch giữa trị đo GNSS và trị thực theo công thức

$$
\Delta_{\mathrm{i}}=\mathrm{S}_{\mathrm{i}}-\mathrm{S}_{\text {thuc }}
$$

Với $\mathrm{S}_{\text {thuc }}$ là chiều dài đoạn thước chuyển dịch (trong thực nghiệm này $S_{\text {thuc }}=10 \mathrm{~mm}$ )

- Đánh giá độ chính xác định vị của công nghệ trạm CORS theo công thức [2]:

$$
\mathrm{m}= \pm \sqrt{\frac{[\Delta \Delta]}{\mathrm{n}}}
$$

Trong đó: $\Delta_{\mathrm{i}}$ là độ lệch của của đoạn đo bằng GNSS và giá trị của đoạn đo theo thang đọc số trên thước chuyên dụng tính theo (6)

n là số đoạn đo kiểm tra trên thực địa

2.2.2. Nội dung thục nghiệm tại khu vục chùa Thầy (Khoảng cách đến trạm tham chiếu là $14,4 \mathrm{~km})$

Nội dung đo thực nghiệm tại khu vực chùa Thầy - Quốc Oai được tiến hành tương tự như mục 2.2.1 nhưng khoảng cách từ điểm thu tín hiệu đến trạm tham chiếu xa hơn - 14,4 km.

\section{Kết quả đo đạc và tính toán thực

\begin{tabular}{|c|c|c|c|c|c|c|}
\hline $\mathrm{i}$ & $\mathrm{k}$ & $\mathrm{X}_{\mathrm{k}(\mathrm{m})}$ & $\mathrm{Y}_{\mathrm{k}(\mathrm{m})}$ & $\mathrm{H}_{\mathrm{k}}(\mathrm{m})$ & & $\mathrm{t}_{\mathrm{i}}(\mathrm{s})$ \\
\hline \multirow{5}{*}{1} & 1 & 2320891.424 & 578466.859 & 7.774 & RTK fix & 2021Y02M25D08H55M00S \\
\hline & 2 & 2320891.426 & 578466.860 & 7.775 & RTK fix & 2021Y02M25D08H55M01S \\
\hline & ........ & & & & & \\
\hline & 298 & 2320891.423 & 578466.863 & 7.773 & RTK fix & 2021Y02M25D08H59M57S \\
\hline & 299 & 2320891.424 & 578466.862 & 7.777 & RTK fix & 2021Y02M25D08H59M58S \\
\hline \multirow{4}{*}{2} & 1 & 2320891.437 & 578466.858 & 7.763 & RTK fix & 2021Y02M25D09H01M00S \\
\hline & 2 & 2320891.433 & 578466.855 & 7.756 & RTK fix & 2021Y02M25D09H01M01S \\
\hline & $\ldots \ldots \ldots$ & ...1. & ............. & $\ldots \ldots \ldots \ldots$ & ...... & \\
\hline & 298 & 2320891.428 & 578466.856 & 7.745 & RTK fix & 2021Y02M25D09H05M57S \\
\hline
\end{tabular} nghiệm}

3.1. Kết quả đo thực nghiệm bằng công nghệ trạm CORS tại khu vực công viên thiên văn có 9 vị trí thu tín hiệu được trình bày tóm tắt trong bảng 1

Bảng 1: Kết quả đo bằng công nghệ trạm CORS tại công viên thiên văn 
Nghiên cứu - Úng dụng

\begin{tabular}{|c|c|c|c|c|c|c|}
\hline & 299 & 2320891.426 & 578466.855 & 7.746 & RTK fix & 2021Y02M25D09H05M58S \\
\hline (................ & ......... & (..................... & ................... & ....... & ................ & \\
\hline
\end{tabular}

3.2. Tiến hành xử lý số liệu đo GNSS theo các công thức (1) đến (6) ta có kết quả như bảng 2

Bảng 2: Kết quả xủ lý số liệu đo GNSS-RTK

\begin{tabular}{|c|c||c||c||c||c||c||}
\hline \hline $\mathrm{i}$ & $\mathrm{X}_{\mathrm{i}}(\mathrm{m})$ & $\mathrm{Y}_{\mathrm{i}}(\mathrm{m})$ & $\Delta \mathrm{X}_{\mathrm{i}}(\mathrm{m})$ & $\Delta \mathrm{Y}_{\mathrm{i}}(\mathrm{m})$ & $\mathrm{S}_{\mathrm{i}}$ đo $(\mathrm{m})$ & $\Delta_{\mathrm{i}}(\mathrm{m})$ \\
\hline \hline 1 & 2320891.423 & 578466.861 & & & & \\
\hline \hline 2 & 2320891.434 & 578466.858 & 0.010 & -0.003 & 0.0108 & 0.0008 \\
\hline \hline 3 & 2320891.432 & 578466.851 & -0.002 & -0.006 & 0.0066 & -0.0034 \\
\hline \hline 4 & 2320891.442 & 578466.846 & 0.010 & -0.005 & 0.0112 & 0.0012 \\
\hline 5 & 2320891.452 & 578466.847 & 0.010 & 0.001 & 0.0098 & -0.0002 \\
\hline 6 & 2320891.459 & 578466.846 & 0.007 & -0.001 & 0.0070 & -0.0030 \\
\hline \hline 7 & 2320891.467 & 578466.842 & 0.008 & -0.003 & 0.0089 & -0.0011 \\
\hline 8 & 2320891.476 & 578466.838 & 0.009 & -0.004 & 0.0097 & -0.0003 \\
\hline \hline 9 & 2320891.486 & 578466.832 & 0.010 & -0.006 & 0.0121 & 0.0021 \\
\hline \hline
\end{tabular}

Trong bảng 2: Giá trị $\Delta_{\mathrm{i}}$ tính theo công thức (Khoảng cách đên trạm tham chiếu là 7,8 km) đạt (6) với $\mathrm{S}_{\text {thuc }}=0.010 \mathrm{~m}$

3.3. Đánh giá độ chính xác định vị bằng công nghệ trạm CORS theo công thức (7) ta có

$$
\mathrm{m}= \pm 0.0019(\mathrm{~m})
$$

hay độ chính xác định vị bằng công nghệ trạm độ chính xác

$$
\mathrm{m}_{\mathrm{p}}= \pm 1.9 \mathrm{~mm}
$$

3.4. Kết quả đo thực nghiệm tại khu vực chùa Thày - Quốc Oai (Khoảng cách đến trạm tham chiếu là 14,4 km)

CORS tại điểm thu tín hiệu - công viên thiên văn

Bảng 3: Kết quả xủ lý số liệu đo bằng công nghệ trạm CORS tại khu vực chùa Thầy

\begin{tabular}{||c|c|c|c|c|c|c||}
\hline $\mathrm{i}$ & $\mathrm{X}_{\mathrm{i}}(\mathrm{m})$ & $\mathrm{Y}_{\mathrm{i}}(\mathrm{m})$ & $\Delta \mathrm{X}_{\mathrm{i}}(\mathrm{m})$ & $\Delta \mathrm{Y}_{\mathrm{i}}(\mathrm{m})$ & $\mathrm{S}_{\mathrm{i}}$ đo $(\mathrm{m})$ & $\Delta_{\mathrm{i}}(\mathrm{m})$ \\
\hline 1 & 2324514.279 & 567341.991 & & & & \\
\hline 2 & 2324514.272 & 567341.994 & -0.007 & 0.003 & 0.0074 & -0.0026 \\
\hline 3 & 2324514.263 & 567342.002 & -0.010 & 0.009 & 0.0130 & 0.0030 \\
\hline 4 & 2324514.252 & 567342.005 & -0.011 & 0.003 & 0.0115 & 0.0015 \\
\hline 5 & 2324514.246 & 567342.013 & -0.006 & 0.008 & 0.0096 & -0.0004 \\
\hline 6 & 2324514.238 & 567342.015 & -0.008 & 0.002 & 0.0085 & -0.0015 \\
\hline 7 & 2324514.229 & 567342.020 & -0.009 & 0.005 & 0.0103 & 0.0003 \\
\hline 8 & 2324514.224 & 567342.021 & -0.005 & 0.001 & 0.0051 & -0.0049 \\
\hline 9 & 2324514.212 & 567342.027 & 0.012 & 0.006 & 0.0133 & 0.0033 \\
\hline \hline
\end{tabular}

Sử dụng công thức (7) để đánh giá độ chính xác ta có: Sai số trung phương định vị điểm đo bằng công nghệ trạm CORS tại khu vực chùa Thày là

$$
\mathrm{m}_{\mathrm{p}}= \pm 2.7 \mathrm{~mm}
$$

Từ kết quả đo đạc và tính toán thực nghiệm cho thấy
- Độ chính xác tương hỗ định vị điểm đơn bằng công nghệ trạm CORS trong thực nghiệm đạt kết quả tương đối tốt so với một số kết quả nghiên cứu truớc đây $[1,2,5,6,7]$, điều này có thể lý giải do khoảng cách đến trạm tham chiếu tương đối ngắn $(7-14 \mathrm{~km})$ và thời gian thu tín hiệu tại mỗi vị trí đo đạt đủ số lượng trị đo cần thiết 
( $\approx 300$ trị đo). Cơ sở khoa học của vấn đề này đã được chúng tôi chứng minh trong tài liệu [4].

- Vì sử dụng hệ thống máy thu GNSS [8] nên ta có thể thu được tín hiệu vệ tinh từ các hệ thống vệ tinh GPS, GLONASS, GALIEO, COMPASS nên số vệ tính luôn đủ để đảm bảo các yêu cầu kỹ thuật định vị bằng công nghệ trạm CORS với yêu cầu độ chính xác cao $(9$ - 14 vệ tinh), đáp ứng được các yêu cầu kỹ thuật cần thiết cho đặc điểm địa hình và điều kiện thời tiết của khu vực cần nghiên cứu.

\section{Kết luận và kiến nghị}

- Có thể sử dụng công nghệ trạm CORS để xác định tọa độ tức thời của điểm quan sát (yêu cầu độ chính xác cao) liên tục, theo thời gian thực và trong mọi điều kiện thời tiết.

- Công nghệ trạm CORS hoàn toàn đáp ứng được các yêu cầu kỹ thuật cần thiết khi theo dõi hiện tượng trượt lở đất đá, sự chuyển vị của các công trình xây dựng do ảnh hưởng của hiện tượng biến đổi khí hậu ở nước ta gây ra.

- Cần tiếp tục khảo sát độ chính xác, nghiên cứu các giải pháp kỹ thuật nhằm nâng cao khả năng ứng dụng và tính hiệu quả của công nghệ trạm CORS trong các môi trường sát với thực tế, phù hợp với đặc điểm khí hậu và điều kiện địa chất của các khu vực có nguy cơ xảy ra hiện tượng trượt lở ở Việt Nam.O

\section{Tài liệu tham khảo}

[1]. Nguyễn Xuân Hoàng (2018), Nghiên cưu ưng dụng công nghệ GPS động và trạm
CORS trong đo đạc địa chính ở Việt Nam, luận văn thạc sĩ kỹ thuật, Trường đại học Mỏ - Địa chất, Hà Nội

[2]. Trần Viết Tuấn, Nguyễn Quang Thắng (2018), Úng dụng công nghệ trạ Cors trong trắc địa công trình, Bài giảng cao học chuyên ngành kỹ thuật trắc địa - bản đồ, Trường Đại học Mỏ - Địa chất, Hà Nội.

[3]. Trần Viết Tuấn, Diêm Công Trang (2018), Khảo sát độ chính xác công nghệ GNSS - RTK trong một số dạng công tác trắc địa công trình", Tạp chí khoa học đo đạc và bản đồ, (37), tr. 46 - 49.

[4]. Diêm Công Trang (2021), Nghiên cúu giải pháp kỹ thuật nâng cao hiệu quả công tác trắc địa trong thi công nhà siêu cao tầng ở Việt Nam, Luận án Tiến sĩ kỹ thuật, Trường Đại học Mỏ - Địa chất, Hà Nội.

[5]. Muhammad Muneeb Shaikh, Syed Zahid Jamal (2014), CORS network for disaster management and emergency response.

[6]. Tomas Soles, Book " CORS and OPUS for Engineers", Journal of surrveying engineering national geodetic survey.

[7]. Ayhan CEYLAN (2018), "Cors networks and investigation of positioning accuracy of Konya permanent GNSS network (Kosaga)", FIG Congress 2018, Istanbul, Turkey.

[8]. Trimble R8s GNSS system, Trimble Navigation Limited USA (2018). $\mathrm{O}$

\section{Summary}

Investigation of the accuracy of cors for some applications of the high precision geodetic works

Tran Viet Tuan, Vietnam Association of Geography, Cartography

\section{Nguyen Van Hiep, Viet My surveying and construcction co.,ltd}

The paper presents the investigation of the accuracy of the Continuously Operation Reference Station for the single point positioning in Vietnam's condition. The method, data experiment, and capability assessment of CORS are introduced in some geodetic work with high precision in Vietnam. O 